\title{
Microwave scattering coefficient of snow in MEMLS and DMRT-ML revisited: the relevance of sticky hard spheres and tomography-based estimates of stickiness
}

\author{
H. Löwe ${ }^{1}$ and G. Picard ${ }^{2,3}$ \\ ${ }^{1}$ WSL Institute for Snow and Avalanche Research SLF, Davos, Switzerland \\ ${ }^{2}$ University Grenoble Alpes, LGGE UMR5183, 38041 Grenoble, France \\ ${ }^{3}$ CNRS, LGGE UMR5183, 38041 Grenoble, France
}

Correspondence to: H. Löwe (loewe@slf.ch)

Received: 11 March 2015 - Published in The Cryosphere Discuss.: 17 April 2015

Revised: 9 October 2015 - Accepted: 13 October 2015 - Published: 16 November 2015

\begin{abstract}
The description of snow microstructure in microwave models is often simplified to facilitate electromagnetic calculations. Within dense media radiative transfer (DMRT), the microstructure is commonly described by sticky hard spheres (SHS). An objective mapping of real snow onto SHS is however missing which prevents measured input parameters from being used for DMRT. In contrast, the microwave emission model of layered snowpacks (MEMLS) employs a conceptually different approach, based on the twopoint correlation function which is accessible by tomography. Here we show the equivalence of both electromagnetic approaches by reformulating their microstructural models in a common framework. Using analytical results for the twopoint correlation function of hard spheres, we show that the scattering coefficient in both models only differs by a factor which is close to unity, weakly dependent on ice volume fraction and independent of other microstructural details. Additionally, our analysis provides an objective retrieval method for the SHS parameters (diameter and stickiness) from tomography images. For a comprehensive data set we demonstrate the variability of stickiness and compare the SHS diameter to the optical equivalent diameter. Our results confirm the necessity of a large grain-size scaling when relating both diameters in the non-sticky case, as previously suggested by several authors.
\end{abstract}

\section{Introduction}

Microwave modeling of snow is commonly addressed within multilayer approaches to account for the vertical layer structure of a snowpack. Examples are HUT (Pulliainen et al., 1999), MEMLS (Wiesmann and Mätzler, 1999; Mätzler and Wiesmann, 1999), DMRT-ML (Picard et al., 2013), and DMRT-QMS (Chang et al., 2014). A similarity of the models is their common approach of applying a one-dimensional radiative transfer scheme to a layered medium. Each layer is assumed to be a statistically homogeneous chunk of snow, in which electromagnetic properties, such as effective permittivity, scattering and absorption coefficient and phase function, are determined by the microstructure. However, the models differ significantly in both the calculation of the solution of the radiative transfer equation and the relation between snow microstructure and the electromagnetic properties. In-depth comparisons of microwave models are hampered by these fundamental differences which remain a source of uncertainty (Tedesco and Kim, 2006). The differences in the representation of snow microstructure led to an open discussion about the appropriate choice of structural metrics for microwave modeling (Brucker et al., 2010). Given the crucial role of a grain size for scattering in snow, the difficulties in comparing different metrics of grain size strongly hinder necessary developments to improve current retrieval schemes (Rott et al., 2010). 
Electromagnetic properties of single snow layers can be theoretically obtained by homogenization methods, if snow is treated as a random, two-phase medium which is statistically homogeneous. The microwave emission model of layered snowpacks (MEMLS) is based on the improved Born approximation (IBA) (Mätzler, 1998) which expresses the scattering coefficient in terms of the Fourier transform of the two-point correlation function. The characterization of the structure in terms of the two-point correlation function $C(r)$ is appealing for random materials, since it naturally emerges in rigorous approaches to effective material properties (Torquato, 2002). The connection between $C(r)$ and the scattering coefficient dates back to the seminal work by Debye and Bueche (1949), which considered general aspects of scattering in random materials. From that perspective, the microstructure representation in IBA appears to be generic. Practically, however, entire correlation functions can hardly be used as "parameters" and further simplifications are commonly required. In MEMLS, IBA is evaluated by assuming an exponential functional form for the correlation function (Debye et al., 1957). As an advantage of this simplification, the microstructure model reduces to a single parameter, the exponential correlation length. This parameter can be conveniently fitted from micro-computed tomography $(\mu \mathrm{CT})$ data (Löwe et al., 2013). Though an exponential correlation function is a reasonable first guess, in particular for depth hoar (Mätzler, 2002), the validity of a single length scale approach for snow must be generally questioned (Löwe et al., 2011). In addition, a rapid retrieval of the exponential correlation length from field measurements is still difficult; only one method was hitherto put forward by Proksch et al. (2015). It is not even clear that three-dimensional microstructures with an exponential correlation function actually exist, and how realizations of such a medium can be generated (Yeong and Torquato, 1998). Generating realizations of a microstructure is however mandatory to compute the scattering from numerical solutions of Maxwell's equations in order to test the IBA assumptions.

Advantages and disadvantages of IBA have to be discussed at eye level of those inherent to another electromagnetic approach to scattering in snow, namely dense media radiative transfer (DMRT). Originally DMRT was developed for random media consisting of spheres or spheroids. Sphere models are attractive from various perspectives. First, the scattering coefficient of sphere assemblies can be calculated analytically in various approximation schemes, such as the quasi-crystalline approximation (QCA), which can be optionally improved by the so-called coherent potential (QCACP) (Tsang and Kong, 2001, Ch. 5). Second, microstructure realizations of spheres can be readily generated to compute the numerical solutions of Maxwell's equations ( $\mathrm{Xu}$ et al., 2010). Third, sphere models have successfully been used for optical properties of snow for a long time (Warren, 1982). An equivalent sphere can always be defined from the specific surface area (SSA) via the optical equivalent diameter.
In addition, the SSA can be rapidly measured in the field by various techniques (Matzl and Schneebeli, 2006; Painter et al., 2007; Gallet et al., 2009; Arnaud et al., 2011). However, even for a hypothetical material consisting of perfect spheres, the sphere diameter and the volume fraction do not characterize the medium completely. Relevant for the scattering of waves are the relative positions of the spheres which determine the relative phases in the superposition of the scattered waves from individual scatterers. Of special importance for snow is the sticky hard sphere (SHS) model due to its ability to change relative particle positions while leaving diameter and volume fraction constant. The concept of SHS was introduced in the context of molecular fluids (Baxter, 1968) and consists of spherical particles interacting via hard-core repulsion and an attractive surface adhesion. In the thermodynamic framework, this competition of attractive and repulsive forces gives rise to a minimal model for a liquid-gas phase transition. For other applications it is appealing to use the thermodynamic equilibrium states for the particle positions as a means of generating microstructures with interesting structural properties; the contact adhesion, which is inversely proportional to the so-called stickiness parameter $\tau$ (Baxter, 1968), gives rise to clustering of the spheres. The relevance of clustering can be directly observed for some snow types, e.g., clustered rounded grains (Fierz et al., 2009). For other snow types the strength of the adhesion must be regarded as a parametric approach to subsume effects of sintering which causes ice crystals to be sticky. The relevance of sticking and the implication on structural properties for new snow has been demonstrated by Löwe et al. (2007). The potential of stickiness for snow microwave modeling to account for relevant length scales beyond a "grain size" was initiated for DMRT by Tsang et al. (2007).

Objective means of estimating the stickiness parameter for a given snow sample are hitherto missing. However, simply resorting to the non-sticky case causes other difficulties. Recent DMRT-based microwave emission modeling (Brucker et al., 2010; Roy et al., 2013; Dupont et al., 2014) and comparison with measurements indicate that the measured optical equivalent diameter can only be used directly as input, if either a "grain-size scaling" (with a factor ranging from 1 to 3 ) is introduced in the non-sticky case or if the stickiness is used as a free, unknown parameter (Roy et al., 2013). This issue could not be further investigated as long as the stickiness parameter $\tau$ remains inaccessible from field measurements. In the absence of objective parameter estimates, a single value is commonly used for all snow types in the simulations (Picard et al., 2013; Tsang et al., 2007), although a large sensitivity of scattering properties on $\tau$ was acknowledged. Due to the lack of interpretation of stickiness for snow, attempts to intercompare DMRT-ML with MEMLS such as Tian et al. (2010) remain challenging, since different microstructural models are in fact used in the respective microwave models. 
It is the aim of the present paper to advance the understanding of microstructural models in different electromagnetic models for microwave modeling of snow by establishing a rigorous link between the scattering formulations used in DMRT-ML and MEMLS. More precisely, for the DMRT theory we consider the QCA-CP approximation as used in DMRT-ML (Picard et al., 2013). For IBA, we follow the derivation based on the internal field factor for spherical scatterers (Mätzler, 1998) which slightly differs from the default implementation in MEMLS that is based on an empirical factor (Mätzler and Wiesmann, 1999). In addition, for both theories we restrict ourselves to small sizes of scatterers relative to the wavelength, which is referred to as a low frequency assumption in the following. By restating the scattering coefficient in both electromagnetic theories and applying established theoretical results for correlation functions in random media, we obtain a rigorous relation between IBA and QCA-CP for arbitrary hard sphere models. On the one hand, this yields an objective method of estimating the stickiness parameter $\tau$ of the SHS model from $\mu \mathrm{CT}$ images of snow. On the other hand, the procedure allows exactly the same microstructural model to be used, namely SHS, in IBA and QCA-CP, and compares the scattering coefficient quantitatively using realistic values for the parameters.

The paper is organized as follows. In Sect. 2 we rederive the scattering coefficient of IBA and QCA-CP to contrast their representation of snow microstructure in terms of different correlation functions. In Sect. 3 we give an explicit expression for the two-point correlation function for SHS which allows the IBA theory to be expressed in terms of the microstructure representation traditionally used by DMRT. In addition, an objective retrieval method of the SHS parameters (diameter and stickiness) from measurements of the two-point correlation function of snow is presented. In Sect. 4 we use existing $\mu \mathrm{CT}$ images to derive the SHS parameters and explore their behavior for different snow types. We discuss our findings in Sect. 5 in view of the relevance of the results for the scattering coefficient in their host models, MEMLS and DMRT-ML. As an application of our work besides the microwave context, we also discuss implications of the present results in view of the discrete element modeling (DEM) of snow for mechanical applications (Johnson and Hopkins, 2005). Presently, DEM also lacks efficient means of objectively reconstructing snow samples in terms of particle models. The reconstruction of snow microstructure in terms of SHS provides a new link to such a granular viewpoint.

\section{Scattering coefficient and relation to microstructure}

\subsection{Two-phase media and two-point correlation functions}

Random two-phase media are a natural starting point to characterize the morphology of air and ice in snow. We consider a two-phase medium in a region $\Omega$ where phases 1 and 2 occupy the subregions $\Omega_{1} \subset V$ with volume $V_{1}$ and $\Omega_{2} \subset V$ with volume $V_{2}$, respectively. Both subvolumes add up to the total volume $V_{1}+V_{2}=V$. We assume phase 1 to be the void phase (i.e., air) and phase 2 the inclusion phase (i.e., ice).

Following Torquato (2002), a single realization of the microstructure can be fully described by the phase indicator function of either phase $j=1,2$ :

$\phi_{j}(\boldsymbol{x})= \begin{cases}1 & \text { if } \boldsymbol{x} \in \Omega_{j} \\ 0 & \text { otherwise },\end{cases}$

which provides the complete information if a point $\boldsymbol{x} \in \Omega$ is covered by ice or air. A binary image obtained by processing $\mu \mathrm{CT}$ data is actually a discrete form of indicator function. From a theoretical point of view, individual realizations are not of particular interest for random media. It is rather the statistical properties which emerge in the analytical derivation of effective physical properties. Volume fractions $\phi_{j}$ of either phase $j=1,2$ are the simplest statistical properties which are first-order quantities, i.e., they just contain the first moment of the indicator function

$\overline{\phi_{j}(\boldsymbol{x})}=\frac{1}{V} \int_{V} \mathrm{~d} \boldsymbol{r} \phi_{j}(\boldsymbol{x})=\frac{V_{j}}{V}=\phi_{j}$,

where volume averaging is denoted by $\bullet$. Snow density is directly related to $\phi_{j}$. In contrast, higher-order moments of the indicator function, i.e., averages of certain products of $\phi_{j}(\boldsymbol{x})$ for $j=1,2$, characterize spatial fluctuations of the phases. The simplest moments are the two-point correlation functions

$S_{j}(\boldsymbol{r})=\overline{\phi_{j}(\boldsymbol{x}+\boldsymbol{r}) \phi_{j}(\boldsymbol{x})}$

of either phase. A closely related, second-order quantity is the phase covariance

$C(\boldsymbol{r})=S_{1}(\boldsymbol{r})-\phi_{1}^{2}=S_{2}(\boldsymbol{r})-\phi_{2}^{2}$,

which is symmetric under the exchange of ice and air. Equations (3) and (4) provide comparable information about a random field that the second moment and the variance provide for a random variable. In practice, second-order moments contain information about the size of heterogeneities at the microscopic scale which can be used to define different "grain sizes" for a given microstructure.

Though snow is known to be anisotropic (Löwe et al., 2013), the IBA and QCA-CP theory have only considered isotropic correlation functions and randomly oriented snow particles up to now. In the following we thus focus on isotropic media, where $C(\boldsymbol{r})=C(r)$ with $r=|\boldsymbol{r}|$. 


\subsection{Scattering coefficient in MEMLS: improved Born approximation}

\subsubsection{Scattering coefficient and phase function}

Below we state the governing equations for the scattering coefficient in the improved Born approximation (Mätzler, 1998). We slightly adapt the notation and terminology to establish the connection to DMRT.

Within the improved Born approximation, the scattering coefficient is derived from the phase function (or bistatic scattering function) (Mätzler, 1998, cf. Eq. 25) which is given by

$\gamma\left(\hat{\boldsymbol{k}}_{\mathrm{s}}, \hat{\boldsymbol{k}}_{\mathrm{i}}\right)=\phi_{2}\left(1-\phi_{2}\right)\left(\varepsilon_{2}-\varepsilon_{1}\right)^{2} K^{2} k_{0}^{4} \sin ^{2}(\chi) I\left(\left|\boldsymbol{k}_{\mathrm{d}}\right|\right)$,

where the dielectric constants of the two phases are denoted by $\varepsilon_{1}$ and $\varepsilon_{2}$. The vectors $\hat{\boldsymbol{k}}_{\mathrm{i}}, \hat{\boldsymbol{k}}_{\mathrm{s}}$ are the propagation directions of the incident and scattered waves, respectively. The angle between the incident electric field and the scattering direction $\hat{\boldsymbol{k}}_{\mathrm{s}}$ is denoted by $\chi$. The wavevector difference of incoming and scattered waves is denoted by $\boldsymbol{k}_{\mathrm{d}}=k_{\mathrm{eff}}\left(\hat{\boldsymbol{k}}_{\mathrm{s}}-\hat{\boldsymbol{k}}_{\mathrm{i}}\right)$ with magnitude

$k_{\mathrm{d}}=2 k_{\mathrm{eff}} \sin (\Theta / 2)$;

hence the angle $\Theta$ denotes the scattering angle, i.e., the angle between $\hat{\boldsymbol{k}}_{\mathrm{s}}$ and $\hat{\boldsymbol{k}}_{\mathrm{i}}$. In Eq. (6), $k_{\mathrm{eff}}$ is the effective propagation constant of the wave in the medium which is related to the effective dielectric constant $\varepsilon_{\text {eff }}$ by

$k_{\text {eff }}=k_{0} \varepsilon_{\text {eff }}^{1 / 2}$,

where $k_{0}$ is the vacuum wavenumber. The remaining quantities in Eq. (5) to be specified are $K$ and $I . K$ denotes the mean squared magnitude ratio of incident and internal field in the ice phase. Various formulations for $K$ are given by Mätzler (1998). To make contact with hard spheres later, we focus on spherical heterogeneities, for which $K$ is given by

$K^{2}=\left|\frac{2 \varepsilon_{\mathrm{eff}, 0}^{\mathrm{IBA}}+\varepsilon_{1}}{2 \varepsilon_{\mathrm{eff}, 0}^{\mathrm{IBA}}+\varepsilon_{2}}\right|^{2}$.

Here $\varepsilon_{\text {eff, } 0}^{\mathrm{IBA}}$ is an approximation to the effective dielectric constant. It represents the dielectric constant of the effective medium in the absence of scattering in the very low frequency limit. It is assumed to be given by the Polder-van Santen mixing formula

$$
\begin{aligned}
\varepsilon_{\mathrm{eff}, 0}^{\mathrm{IBA}} & =\frac{2 \varepsilon_{1}-\varepsilon_{2}+3 \phi_{2}\left(\varepsilon_{2}-\varepsilon_{1}\right)}{4} \\
& +\frac{\sqrt{\left(2 \varepsilon_{1}-\varepsilon_{2}+3 \phi_{2}\left(\varepsilon_{2}-\varepsilon_{1}\right)\right)^{2}+8 \varepsilon_{1} \varepsilon_{2}}}{4} .
\end{aligned}
$$

The most relevant quantity in Eq. (5) for the purpose of this paper is $I\left(\left|\boldsymbol{k}_{\mathrm{d}}\right|\right)$ which contains the entire information about the microstructure entering the scattering coefficient in IBA. From the definition of $I\left(\left|\boldsymbol{k}_{\mathrm{d}}\right|\right)$ in Mätzler (1998), we can rewrite it in terms of the Fourier transform of the correlation function Eq. (4) according to

$I\left(\left|\boldsymbol{k}_{\mathrm{d}}\right|\right)=\frac{1}{4 \pi} \frac{\widetilde{C}\left(\left|\boldsymbol{k}_{\mathrm{d}}\right|\right)}{\phi_{2}\left(1-\phi_{2}\right)}$.

Here and throughout we use the shorthand notation $\widetilde{f}$ to indicate the three-dimensional Fourier transform of a function $f$ which is defined by

$$
\tilde{f}(\boldsymbol{k})=\int_{\mathbb{R}^{3}} \mathrm{~d} \boldsymbol{x} f(\boldsymbol{x}) \exp (-i \boldsymbol{x} \cdot \boldsymbol{k}),
$$

which implies the inverse transform

$$
f(\boldsymbol{x})=(2 \pi)^{-3} \int_{\mathbb{R}^{3}} \mathrm{~d} \boldsymbol{k} \tilde{f}(\boldsymbol{k}) \exp (i \boldsymbol{x} \cdot \boldsymbol{k}) .
$$

We close this section by commenting on the ambiguous notion of "particles" in the original derivation of IBA. The field ratio matrix K (Mätzler, 1998, Eq. 7) does not follow on from the theory but needs to be given a priori. As explained by Mätzler and Wiesmann (1999), it depends on snow density and grain shape, stating "the shape dependence is relatively weak; therefore, the real situation can be wellmodeled with idealized particles." It is however questionable that the signature of local shape $(K)$ and the correlation function $C(\boldsymbol{x})$ can be chosen independently, as suggested in IBA, since both quantities are related. A potential impact of shape is however irrelevant for the present isotropic considerations, where we choose $K$ corresponding to spherical particles (Mätzler, 1998, Eq. 28) to be consistent with the DMRT description.

\subsubsection{Scattering coefficient in the low frequency limit}

The scattering coefficient $\kappa_{\mathrm{S}}^{\mathrm{IBA}}$ for IBA in Mätzler (1998) is obtained by integrating the phase function Eq. (5) over the scattering directions $\hat{\boldsymbol{k}}_{\mathrm{s}}$, viz

$\kappa_{\mathrm{S}}^{\mathrm{IBA}}=\frac{1}{4 \pi} \int_{4 \pi} \mathrm{d} \Omega_{\mathrm{s}} \gamma\left(\hat{\boldsymbol{k}}_{\mathrm{s}}, \hat{\boldsymbol{k}}_{\mathrm{i}}\right)$,

where $\mathrm{d} \Omega_{\mathrm{s}}$ is the solid angle element in the scattering direction $\hat{\boldsymbol{k}}_{\mathrm{s}}$. In general, not only $\sin ^{2} \chi$ but also $I\left(\left|\boldsymbol{k}_{\mathrm{d}}\right|\right)$ depends on the scattering angle in Eq. (5) which requires numerical integration of Eq. (12) as done in MEMLS. In the following, we focus on the low frequency limit which allows $I\left(\left|\boldsymbol{k}_{\mathrm{d}}\right|\right)$ to be replaced by $I$ (0) in Eq. (5) as also done by Mätzler (1998). It follows that the only dependence of the bistatic scattering coefficient Eq. (5) on $\hat{\boldsymbol{k}}_{\mathrm{S}}$ remains in the term $\sin ^{2} \chi$. Without loss of generality, choosing a coordinate system where the $z$ axis is aligned with the local field, the average Eq. (12) of $\sin \chi$ yields $2 / 3$. In summary, the scattering coefficient is 
$\kappa_{\mathrm{S}}^{\mathrm{IBA}}=\frac{2}{3} k_{0}^{4}\left|\frac{\left(\varepsilon_{2}-\varepsilon_{1}\right)\left(2 \varepsilon_{\mathrm{eff}, 0}^{\mathrm{IBA}}+\varepsilon_{1}\right)}{\left(2 \varepsilon_{\mathrm{eff}, 0}^{\mathrm{IBA}}+\varepsilon_{2}\right)}\right|^{2} \frac{\widetilde{C}(0)}{4 \pi}$,

where we used Eq. (10) to obtain an expression in terms of the correlation function.

\subsection{Particle-based media and pair correlation functions}

The original versions of DMRT assume that the microstructure comprises $N$ discrete particles of a given shape and size in a container of volume $V$. A particular realization of the particle configuration can be specified in terms of the particle centers $\left\{\boldsymbol{r}_{i}\right\}_{i=1 \ldots N}$. In contrast to IBA, which describes the microstructure in terms of the phase indicator function containing implicit information about shapes and sizes, DMRT requires explicit information about particle shape and size of a particle at locations $\left\{\boldsymbol{r}_{i}\right\}$. Often a fixed shape is chosen (e.g., sphere) and the size is either left constant (referred to as monodisperse) or drawn from a probability distribution (referred to as polydisperse). Thus for a given shape and size, a particular realization of the microstructure can be described by the number density field

$n(\boldsymbol{x})=\sum_{i=1}^{N} \delta\left(\boldsymbol{x}-\boldsymbol{r}_{i}\right)$.

The first moment is the mean number density

$\overline{n(\boldsymbol{x})}=\frac{1}{V} \int_{V} \mathrm{~d} \boldsymbol{x} n(\boldsymbol{x})=\frac{N}{V}=n$.

From the number density field we define a second-order correlation function by

$c(\boldsymbol{r})=\overline{(n(\boldsymbol{x})-n)(n(\boldsymbol{x}+\boldsymbol{r})-n)}$,

which characterizes local fluctuation of the number density. The well-known pair correlation function $g(\boldsymbol{r})$ of a particle assembly is then essentially a modified version of Eq. (16), viz

$c(\boldsymbol{r})=n^{2}[g(\boldsymbol{r})-1]+n \delta(\boldsymbol{r})$.

The structure factor $S(\boldsymbol{k})$ is the Fourier transform of the pair correlation function and given by

$S(\boldsymbol{k})=1+n \int_{V} \mathrm{~d} \boldsymbol{r}[g(\boldsymbol{r})-1] \exp (-i \boldsymbol{r} \cdot \boldsymbol{k})$.

2.4 Scattering coefficient in DMRT-ML: quasi-crystalline approximation - coherent potential and low frequency limit

Several flavors of DMRT have been developed over the years (Tsang, 1992; Tsang et al., 2000, 2007; Liang et al., 2008).
Here we consider dense packings of spheres in the low frequency limit, in accordance with the approximations made in the derivation of IBA. The response of a sphere on an electric field is well-known. To obtain the scattering coefficient the main task is to estimate the collective excitation of all spheres upon an incident plane wave. This can be stated in terms of an integral equation (Tsang and Kong, 2001, Ch. 5) which allows the effective dielectric constant $\varepsilon_{\text {eff }}$ of the medium to be computed. In the quasi-crystalline approximation (QCA) with coherent potential (CP), the result for the effective dielectric constant is given by

$\varepsilon_{\mathrm{eff}}=\varepsilon_{1}+3 \varepsilon_{\mathrm{eff}} \phi_{2} \Lambda\left(\varepsilon_{\mathrm{eff}}\right)\left\{1+i \frac{2}{3} \varepsilon_{\mathrm{eff}}^{3 / 2} a^{3} \Lambda\left(\varepsilon_{\mathrm{eff}}\right) S(0)\right\}$.

Equation (19) is a nonlinear equation for the complex-valued effective dielectric constant $\varepsilon_{\text {eff. }}$. It involves the structure factor $S(\boldsymbol{k})$ from Eq. (18) in the low frequency limit $\boldsymbol{k} \rightarrow 0$, the sphere radius $a$ and the auxiliary function

$\Lambda\left(\varepsilon_{\mathrm{eff}}\right)=\frac{\varepsilon_{2}-\varepsilon_{1}}{3 \varepsilon_{\mathrm{eff}}+\left(\varepsilon_{2}-\varepsilon_{1}\right)\left(1-\phi_{2}\right)}$.

Different strategies are possible to solve Eq. (19). The simplest strategy is to replace $\varepsilon_{\text {eff }}$ on the right-hand side by $\varepsilon_{1}$. This approximation is known as QCA (without coherent potential) (Tsang and Kong, 2001, Eq. 5.3.113a). The solution strategy followed by Picard et al. (2013) is instead iterative. First, Eq. (19) is solved in the non-scattering limit, i.e., for $a=0$. This yields a quadratic equation, cf. (Tsang and Kong, 2001, Eq. 5.3.125) or (Picard et al., 2013, Eq. (5)). We denote the solution of the equation by $\varepsilon_{\text {eff, } 0}^{\mathrm{QCA}-\mathrm{CP}}$ which is given by

$$
\begin{aligned}
& \varepsilon_{\text {eff, } 0}^{\mathrm{QCA}-\mathrm{CP}}=\frac{\varepsilon_{1}-\frac{\left(\varepsilon_{2}-\varepsilon_{1}\right)}{3}\left(1-4 \phi_{2}\right)}{2} \\
& +\frac{\sqrt{\left(\varepsilon_{1}-\frac{\left(\varepsilon_{2}-\varepsilon_{1}\right)}{3}\left(1-4 \phi_{2}\right)\right)^{2}+4 \varepsilon_{1} \frac{\left(\varepsilon_{2}-\varepsilon_{1}\right)}{3}\left(1-\phi_{2}\right)}}{2} .
\end{aligned}
$$

The extinction coefficient can be derived from Eq. (19) via $\kappa_{\mathrm{e}}=k_{0} \mathcal{I}\left(\varepsilon_{\text {eff }}^{1 / 2}\right)$. Following Tsang (1992, Eqs. 46 and 90), the scattering coefficient is given by

$\kappa_{\mathrm{S}}^{\mathrm{QCA}-\mathrm{CP}}=\frac{2}{9} k_{0}^{4} a^{3} \phi_{2}\left|\frac{3 \varepsilon_{\mathrm{eff}, 0}^{\mathrm{QCA}-\mathrm{CP}}\left(\varepsilon_{2}-\varepsilon_{1}\right)}{3 \varepsilon_{\mathrm{eff}, 0}^{\mathrm{QCA}, \mathrm{CP}}+\left(\varepsilon_{2}-\varepsilon_{1}\right)\left(1-\phi_{2}\right)}\right|^{2} S(0)$.

The expression Eq. (22) for the scattering coefficient is generic since it involves the microstructure in terms of the structure factor at low frequency $S(0)$. This can be specified to arbitrary particle systems as long as the structure factor $S(0)$ can be computed.

\section{Link between IBA and QCA-CP}

The statistical characterization of particle systems by the number density field Eq. (14) has a long tradition in the statistical physics of liquids. A comparison with Sect. 2 reveals 
that particle-based media and two-phase media are described by similar, though slightly different formalisms: Both are microscopically defined by a microscopic quantity characterizing the local density - local number density Eq. (14) vs. local volume fraction Eq. (1) - and moments of the microscopic quantities (Eqs. 15, 16 vs. Eqs. 2, 3) for the mean and fluctuations of the microscopic quantities. Differences emerge due to the mathematical properties of the microscopic quantities. While in the two-phase case, products $\phi_{j}(\boldsymbol{r})^{2}$ of the indicator function with itself are well-defined, products of the form $\delta(\boldsymbol{r})^{2}$ are mathematically meaningless. This requires some caution when computing moments.

The interrelation of the statistical description of two-phase media and particle-based media in terms of correlation functions was detailed by Torquato and Stell (1982). To relate the scattering coefficient from IBA and QCA-CP, Eqs. (22) and (13) respectively, we note that one is determined by the low frequency limit of the structure factor $S(0)$, while the other is determined by the low frequency limit $C(0)$ of the Fourier transform of the correlation function. While the former is only defined for particle systems, the latter can be specified to arbitrary two-phase media. Thus for particlebased media, both correlation functions can be defined. The pair correlation function of the particle centers and the twopoint correlation function are not independent, since knowledge of particle positions together with the particle shape uniquely determines the spatial region which is covered by the particle phase (phase 2). This is exactly the information contained in the indicator function.

\subsection{Relation between $g(r)$ and $C(r)$}

The link between corresponding correlation functions was established (Torquato and Stell, 1983) under quite general assumptions for arbitrary particle interactions. The specification of the general result to the case of monodisperse hard spheres can be found in Zachary and Torquato (2009, Eq. 52). In the present notation, the result reads

$$
C(\boldsymbol{r})=n v_{\text {int }}(\boldsymbol{r}, d)+n^{2} v_{\text {int }}(\boldsymbol{r}, d) *[g(\boldsymbol{r})-1],
$$

where $(*)$ denotes the three-dimensional convolution and $v_{\text {int }}$ is the volume of the intersection set of two identical spheres with diameter $d=2 a$ which are separated by $\boldsymbol{r}$. The intersection volume (Torquato, 2002, Eq. 3.51) is given by

$v_{\text {int }}(\boldsymbol{r}, d)=v(d)\left(1-\frac{3}{2} \frac{|\boldsymbol{r}|}{d}+\frac{1}{2} \frac{|\boldsymbol{r}|^{3}}{d^{3}}\right) H(d-|\boldsymbol{r}|)$,

in terms of the Heaviside step function $H(x)$ and the volume of the sphere $v(d)=\pi d^{3} / 6$. In view of the scattering coefficient derivation, we take the Fourier transform of Eq. (23) and use the definition of the structure factor Eq. (18) to obtain

$$
\widetilde{C}(\boldsymbol{k})=n \widetilde{v}_{\text {int }}(\boldsymbol{k}, d) S(\boldsymbol{k}) .
$$

The Fourier transform of the intersection volume Eq. (24) can be readily computed and written in the scaling form

$\widetilde{v}_{\text {int }}(\boldsymbol{k}, d)=v(d)^{2} P(k d)$

with

$P(k d)=\left[\frac{3(\sin (k d / 2)-k d / 2 \cos (k d / 2))}{(k d / 2)^{3}}\right]^{2}$

and $k=|\boldsymbol{k}|$. The function $P(k d)$ is known as the spherical form factor (Pedersen, 1997, Eq. 55). The relation between Fourier transforms of the phase covariance and correlation function of the particle assembly, given by Eq. (25), constitutes the key result to compare the scattering coefficient in IBA and QCA-CP. In addition, this relation would allow any particle-based model to be implemented in IBA, as long as the structure factor $S(0)$ and field ratio $K$ are known. The relation Eq. (23) is not limited to spheres; it can be generalized also to anisotropic particles, provided the intersection volume $v_{\text {int }}$ can be computed.

\subsection{Relation between scattering coefficient in IBA and QCA-CP}

By means of the fundamental relation Eq. (25) and $\phi_{2}=n v(d)$ we can now express the IBA scattering coefficient Eq. (13) in terms of the structure factor, yielding

$\kappa_{\mathrm{s}}^{\mathrm{IBA}}=\frac{2}{9} k_{0}^{4} a^{3} \phi_{2}\left|\frac{\left(\varepsilon_{2}-\varepsilon_{1}\right)\left(2 \varepsilon_{\mathrm{eff}, 0}^{\mathrm{IBA}}+\varepsilon_{1}\right)}{\left(2 \varepsilon_{\mathrm{eff}, 0}^{\mathrm{IBA}}+\varepsilon_{2}\right)}\right|^{2} S(0)$,

which is very close to the form of the QCA-CP scattering coefficient in Eq. (22). For a comparison we define $r_{\mathrm{s}}$ as the ratio of the IBA and QCA-CP scattering coefficient which is given by

$$
\begin{aligned}
r_{\mathrm{S}}\left(\phi_{2}\right) & =\frac{\kappa_{\mathrm{s}}^{\mathrm{IBA}}}{\kappa_{\mathrm{S}}^{\mathrm{QCA}-\mathrm{CP}}} \\
& =\left|\frac{\left(2 \varepsilon_{\mathrm{eff}, 0}^{\mathrm{IBA}}+\varepsilon_{1}\right)\left(3 \varepsilon_{\mathrm{eff}, 0}^{\mathrm{QCA}-\mathrm{CP}}+\left(\varepsilon_{2}-\varepsilon_{1}\right)\left(1-\phi_{2}\right)\right)}{\left(2 \varepsilon_{\mathrm{eff}, 0}^{\mathrm{IBA}}+\varepsilon_{2}\right) 3 \varepsilon_{\mathrm{eff}, 0}^{\mathrm{QCA}-\mathrm{CP}}}\right|^{2}
\end{aligned}
$$

For the given phase permittivities $\varepsilon_{1}, \varepsilon_{2}$ the ratio is only a function of the ice volume fraction $\phi_{2}$. The dependence on the microstructure via $S(0)$ dropped out completely in Eq. (29) since both scattering coefficients contain exactly the same factor. The ratio $r_{\mathrm{s}}$ is also independent of the wavelength (except implicitly through the dielectric constants) and varies by no more than $\approx 30 \%$ for relevant volume fractions $0<\phi_{2}<0.5$ (Fig. 1). This has to be contrasted to the ratio calculated and shown in Mätzler (1998, Fig. 4), in which a strong influence of the volume fraction on $r_{\mathrm{s}}$ is apparent. The reason is the use of different microstructural models (overlapping spheres versus SHS) in the respective scattering models. For completeness, we also show that the real and 


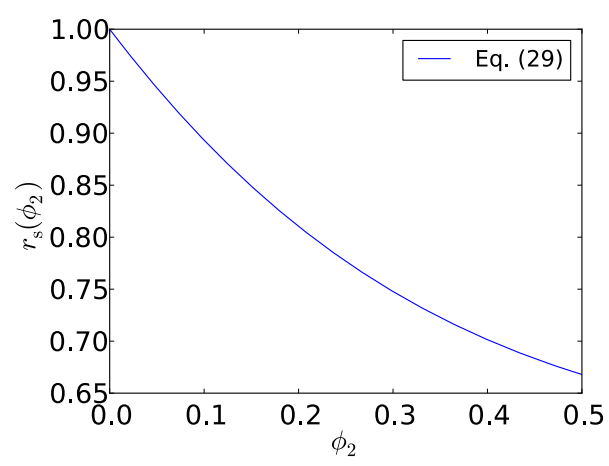

Figure 1. Ratio of scattering coefficient calculated with IBA and DMRT theory as a function of ice volume fraction $\phi_{2}$ evaluated for $\varepsilon_{1}=1$ and $\varepsilon_{2}=3.17+0.0022 i$.

imaginary parts of the dielectric constants of the effective medium in the absence of scattering of either model, Eqs. (9) and (21) in Fig. 2. The maximum relative difference between the real parts is only $1.5 \%$, and between the imaginary parts it is $8.8 \%$.

\subsection{Structure factor for sticky hard spheres}

The DMRT formulation used in many DMRT-based models use the sticky hard sphere (SHS) model to represent the position of the scatterers. In IBA it is now possible to consider the same SHS model since the scattering coefficient Eq. (28) can be specified to an arbitrary sphere assembly as long as the structure factor $S(0)$ is known. To this end we give an expression of the structure factor $S(k)$ for SHS and its zero $k$ limit in order to obtain an IBA-based formulation as close as possible to that of DMRT. We consider the monodisperse SHS model by Baxter (1968) comprising $N$ point particles at positions $\left\{\boldsymbol{r}_{i}\right\}_{i=1 \ldots N}$ in a volume $V$ interacting via a pair potential

$U(|\boldsymbol{r}|)=\frac{1}{\beta} \begin{cases}\infty, & |\boldsymbol{r}|<d \\ -\ln \left[\frac{\sigma}{12 \tau(\sigma-d)}\right], & d<|\boldsymbol{r}|<\sigma . \\ 0, & |\boldsymbol{r}|>\sigma\end{cases}$

Here $\beta$ is the inverse temperature and the potential consists of a hard-core repulsion, which prevents particles from overlap at distances smaller than the sphere diameter $d$, and a square well attraction, which tends to a contact adhesion force in the limit $\sigma \rightarrow d$. The strength of the adhesion is proportional to the inverse of the stickiness parameter $\tau$. In the PercusYevick approximation, the structure factor of SHS can be written in closed form (Tsang et al., 2001, Eqs. 8.4.198.4.22) in terms of the particle phase volume fraction $\phi_{2}$, the stickiness parameter $\tau$, and sphere diameter $d$ according to

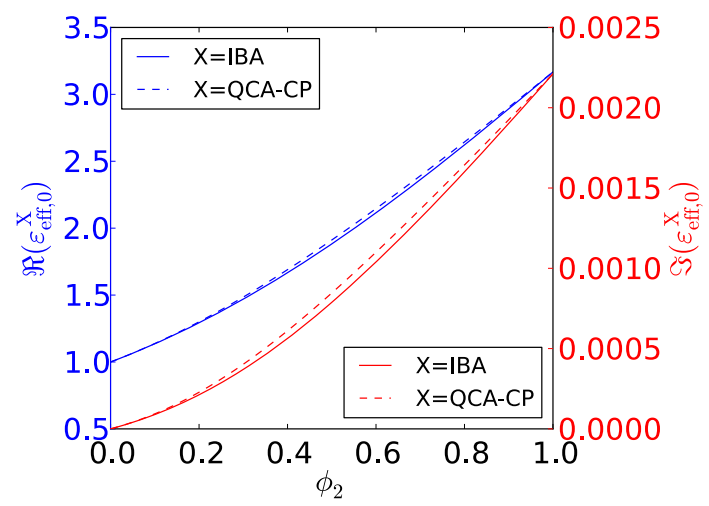

Figure 2. Real and imaginary parts of dielectric constant $\varepsilon_{\mathrm{eff}, 0}^{\mathrm{IBA}}$ and $\varepsilon_{\text {eff, } 0}^{\mathrm{QCA}-\mathrm{CP}}$ in the very low frequency limit as a function of ice volume fraction $\phi_{2}$.

$$
\begin{aligned}
S_{\mathrm{SHS}}(k) & =\left[A(X)^{2}+B(X)^{2}\right]^{-1} \\
A(X) & =\frac{\phi_{2}}{1-\phi_{2}}\left[\left(1-t \phi_{2} \frac{3 \phi_{2}}{1-\phi_{2}}\right) \Phi(X)\right. \\
+ & \left.\left(3-t\left(1-\phi_{2}\right)\right) \Psi(X)\right]+\cos (X) \\
B(X) & =\frac{\phi_{2}}{1-\phi_{2}} X \Phi(X)+\sin (X) \\
\Phi(X) & =3\left[\frac{\sin (X)}{X^{3}}-\frac{\cos (X)}{X^{2}}\right] \\
\Psi(X) & =\frac{\sin (X)}{X},
\end{aligned}
$$

with $X=k d / 2$ and the parameter $t$, given by the smallest solution of the quadratic equation

$$
\frac{\phi_{2}}{12} t^{2}-\left(\tau+\frac{\phi_{2}}{1-\phi_{2}}\right) t+\frac{1+\phi_{2} / 2}{\left(1-\phi_{2}\right)^{2}}=0
$$

under the additional condition $t<\left(1+2 \phi_{2}\right) /\left(\phi_{2}\left(1-\phi_{2}\right)\right)$ which guarantees $S_{\mathrm{SHS}}(0)$ to be positive (Baxter, 1968; Tsang et al., 2001). The structure factor $S(k)$ is thus only a function of the scaling variable $k d$. Using the limiting values $\Psi(0)=\Phi(0)=1$ and $B(0)=0$, the low frequency limit $S(0)$ required for the scattering coefficient can be readily computed from Eq. (31) and is given by

$S(0)=A(0)^{-2}=\left[\frac{\left(1-\phi_{2}\right)^{2}}{1+2 \phi_{2}-t \phi_{2}\left(1-\phi_{2}\right)}\right]^{2}$.

This is in agreement with Picard et al. (2013), Tsang and Kong (2001) and Shih et al. (1997) The structure factor for non-sticky hard spheres can be recovered by taking the limit $\tau \rightarrow \infty$, corresponding to $t \rightarrow 0$, yielding $S(0)=\left(1-\phi_{2}\right)^{4} /\left(1+2 \phi_{2}\right)^{2}$.

In summary, the Fourier transform of the correlation function of sticky hard spheres can be written as

$\widetilde{C}_{\mathrm{SHS}}\left(k \mid \phi_{2}, d, \tau\right)=\phi_{2} v(d) P(k d) S_{\mathrm{SHS}}(k d)$ 
in terms of the sphere volume $v(d)=\pi d^{3} / 6$, the spherical form factor $P(k d)$ from Eq. (27) and the SHS structure factor $S_{\mathrm{SHS}}$ from Eq. (31). For later purposes we made the dependence of $\widetilde{C}_{\mathrm{SHS}}\left(k \mid \phi_{2}, d, \tau\right)$ on the involved parameters $\phi_{2}$, $d, \tau$ explicit.

\section{SHS analysis of snow from $\mu \mathrm{CT}$ images}

\subsection{Micro-computed tomography data}

For the following analysis we employ the data set of $\mu \mathrm{CT}$ images described in Löwe et al. (2013) which was used therein in a different context. The entire data set comprises 167 snow samples which are divided into several series of data: two time series of isothermal experiments (ISO-1, ISO5), four time series of temperature gradient metamorphism experiments (TGM-2, TGM-17, DH-1, DH-2) and a set of 37 uncorrelated samples (DIV) comprising various snow types. A detailed characterization including the IACS international classification of seasonal snow on the ground (Fierz et al., 2009) of the snow samples is given in the supplement to Löwe et al. (2013). In summary, the data sets contains 62 samples of depth hoar (DH), 54 of rounded grains (RG), 33 of faceted crystals (FC), 10 of decomposing and fragmented precipitation particles (DF), 5 of melt forms (MF) and 3 of precipitation particles (PP).

\subsection{Data processing and Fourier transform}

Computing correlation functions as convolutions of $\mu \mathrm{CT}$ images is commonly done using fast Fourier transform (FFT) of the indicator function Eq. (1). Fourier transforms of the data are thus naturally available.

Snow is known to be anisotropic which was explicitly analyzed for the present data set in Löwe et al. (2013). To interpret the anisotropic $\mu \mathrm{CT}$ data in terms of the isotropic SHS model, orientational averaging of the experimental data is required. To this end we cut out maximal cubic subsets of linear size $L$ from the original $\mu \mathrm{CT}$ images to obtain a wavevector spacing $\Delta k=2 \pi / L$ which is independent of coordinate direction in Fourier space. Sample sizes $L$ of the cubic images vary from 4.8 to $10.7 \mathrm{~mm}$, and voxelsizes $l_{\text {vox }}$ from 10 to $54 \mu \mathrm{m}$, respectively. An a posteriori inspection will confirm that $L$ is approximately an order of magnitude larger than the optical diameter for all samples. The angular average $\widetilde{C}(k)$ is obtained by radially binning the threedimensional Fourier transform and averaging over points in concentric wavevector shells. The angular average $\widetilde{C}(k)$ constitutes the experimental data that will be compared to the SHS model $\widetilde{C}_{\text {SHS }}\left(k \mid \phi_{2}, d, \tau\right)$ from Eq. (34).

\subsection{Properties of the SHS correlation function}

Before turning to the parameter estimation, we illustrate the parametric behavior of $\widetilde{C}_{\mathrm{SHS}}\left(k \mid \phi_{2}, d, \tau\right)$ for sticky hard

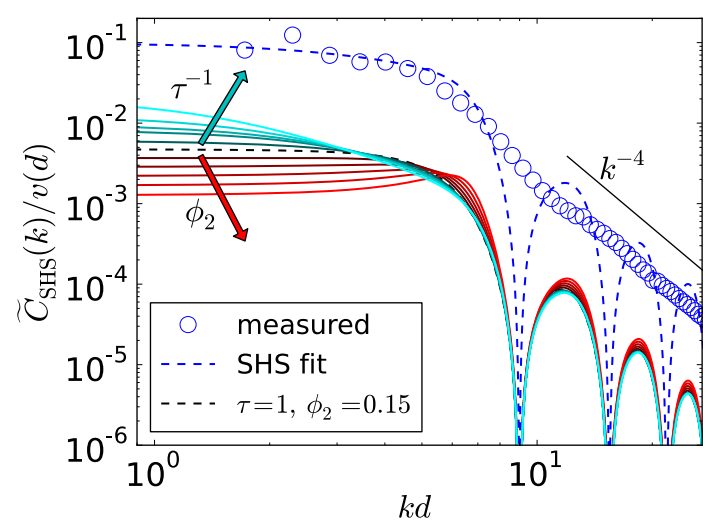

Figure 3. Comparison of measured and fitted Fourier transforms of the correlation function for one snow sample (blue curves). Dependence of sticky hard sphere model $\widetilde{C}_{\text {SHS }}\left(k \mid \phi_{2}, d, \tau\right)$ on stickiness $\tau$ (cyan curves) and volume fraction $\phi_{2}$ (red curves) around a reference (black dashed line). The dependence of $\widetilde{C}_{\mathrm{SHS}}\left(k \mid \phi_{2}, d, \tau\right)$ on sphere diameter $d$ is captured by using the dimensionless variable $k d$ and normalizing with the sphere volume $v(d)$.

spheres. To this end we picked one snow sample of rounded grains and show the experimental data $\widetilde{C}(k)$ together with the best fit of the model in Fig. 3. We also indicated the behavior for large $k$ as a guide to the eye. The Fourier transform of the correlation function must asymptotically decay as $\sim s k^{-4}$, where the prefactor $s$ is the surface area per unit volume of the sample (Torquato, 2002).

To further demonstrate the impact of the parameters $\left(\phi_{2}\right.$, $\tau, d)$ on the SHS model $\widetilde{C}_{\text {SHS }}\left(k \mid \phi_{2}, d, \tau\right)$, we additionally varied the parameters around some reference values $d=1$, $\tau=1$ and $\phi_{2}=0.15$ (black dashed line in Fig. 3). One family of curves (red colors) illustrates the impact of increasing volume fraction $\phi_{2}$ by plotting the curves $d=1, \tau=1$ and $\phi_{2}=[0.2,0.25,0.3,0.35,0.4]$. The other family of curves (cyan colors) illustrates the impact of increasing stickiness $\tau^{-1}$ by plotting the curves $d=1, \tau=[0.5,0.3,0.25,0.2$, $0.15]$ and $\phi_{2}=0.15$. The dependence on sphere diameter is fully captured by using a non-dimensionalized scaling plot $\widetilde{C}(k) / v(d)$ vs. $k d$ as predicted by Eq. (34). The red and cyan curves were multiplied by an arbitrary factor to vertically translate the curves for better visibility.

\subsection{SHS parameter estimation for snow}

Using the closed form expression $\widetilde{C}_{\mathrm{SHS}}\left(k \mid \phi_{2}, d, \tau\right)$ for the correlation function from Eq. (34) we are now able to objectively estimate optimal SHS parameters $\hat{d}$ and $\hat{\tau}$ for a given snow sample by fitting the expression to the angularaveraged experimental data $\widetilde{C}(k)$. The volume fraction $\phi_{2}$ is prescribed by the value obtained from the $\mu \mathrm{CT}$ image. We used the same fit interval $k=\left[0, k_{\max } / 3\right]$ for all samples. The number of points in this interval and the maximum wavevector $k_{\max }=2 \pi / l_{\mathrm{vox}}$ however varies from sample to 


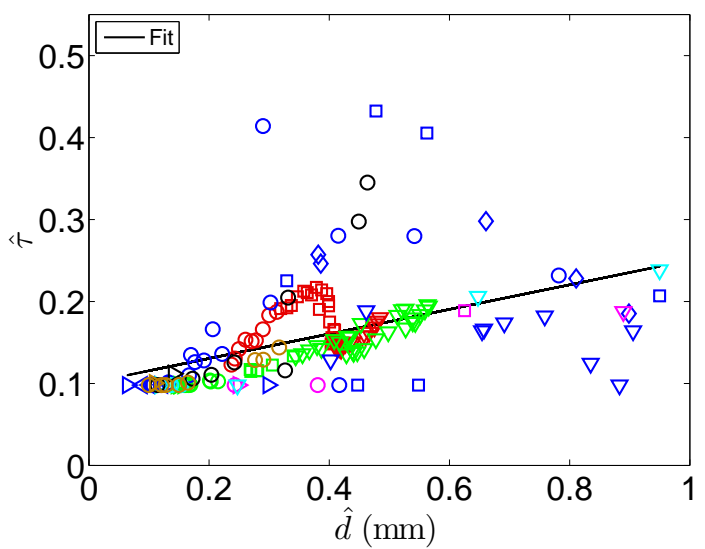

Figure 4. Estimated parameter pairs $(\hat{d}, \hat{\tau})$ for all 167 samples. Experiment series are indicated by colors (TGM-17: red, TGM2: green, DIV: blue, DH-1: magenta, DH-2: cyan, ISO-1: black, ISO-5: brown) and snow types by markers (PP: $\triangleleft, \mathrm{DF}: \triangleright, \mathrm{RG}: \circ$, FC: $\square, \mathrm{DH}: \vee, \mathrm{MF}: \diamond)$.

sample due to variations in voxelsize $l_{\text {vox }}$ and sample size $L$ of the cubic $\mu \mathrm{CT}$ image. The regression on $d, \tau$ was carried out using MATLAB's nonlinear, least-squares fitting tools to minimize the sum of squared differences between model and measurement. Thereby, we maximize the quality of fit in the low $k$ regime, since the scattering depends only on the value at the origin $k d=0$. First we present overview results for the optimal parameters; further details such as goodness of fits and characteristics of the cost function are explored in the subsequent section.

For an overview, we show the optimal parameters $(\hat{d}, \hat{\tau})$ for all samples in Fig. 4. The fit parameters are apparently unrelated; a linear regression $\hat{\tau}=0.15 \hat{d}+0.10$ as an attempt to predict stickiness from the sphere diameter (black line) yields a coefficient of determination of $R^{2}=0.23$.

Next we plot the optimal stickiness values $\hat{\tau}$ for all 167 samples in Fig. 5 as a function of ice volume fraction. This plot corresponds to the thermodynamic phase diagram of the original SHS model which completely determines the physical behavior of the system. It allows the distribution of pairs $\left(\phi_{2}, \hat{\tau}\right)$ to be assessed further. Two additional lines are added in the Fig. 5. The dashed line indicates the lower limit of physically admissible values of $\tau$ for a given volume fraction. The line is given by

$\tau_{\min }\left(\phi_{2}\right)=\frac{1}{12} \frac{14 \phi_{2}^{2}-4 \phi_{2}-1}{2 \phi_{2}^{2}-\phi_{2}-1}$

as an implication of the condition on $t$ in Eq. (32).

The second (full) line in Fig. 5, which separates the parameter space into a white and a gray part, corresponds to the underlying percolation transition of the SHS model. The percolation line is given by

$\tau_{\text {perc }}\left(\phi_{2}\right)=\frac{1}{12} \frac{19 \phi_{2}^{2}-2 \phi_{2}+1}{\left(1-\phi_{2}\right)^{2}}$

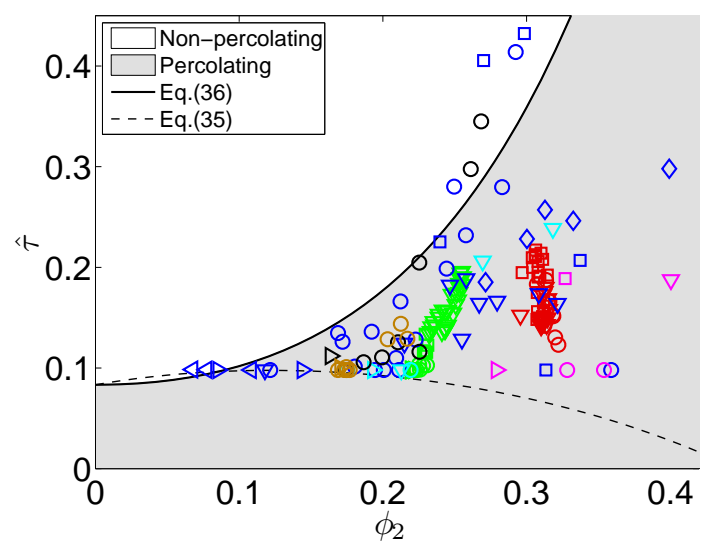

Figure 5. Estimated stickiness values $\hat{\tau}$ as a function of ice volume fraction $\phi_{2}$. Experiment series are indicated by colors (TGM17: red, TGM-2: green, DIV: blue, DH-1: magenta, DH-2: cyan, ISO-1: black, ISO-5: brown) and snow types by markers (PP: $\triangleleft$, DF: $\triangleright$, RG:, FC: $\square, \mathrm{DH}: \vee, \mathrm{MF}: \diamond)$.

cf. Chiew and Glandt (1983). The relevance of both lines in view of the present application is further detailed in the discussion.

Finally we compare the estimated SHS diameter $\hat{d}$ with the optical diameter $d_{\text {opt }}$. The optical diameter of a snow sample is defined by

$d_{\text {opt }}=\frac{6}{\operatorname{SSA} \rho_{\text {ice }}}$

in terms of the SSA which was obtained from the $\mu \mathrm{CT}$ image. The results are shown in Fig. 6. The relation between both diameters will be further analyzed in the context of grain-size scaling below.

\subsection{Goodness of fits}

To illustrate differences in the performance of the fit for the SHS model, we show the coefficient of determination $R^{2}$ for the optimal values $\hat{\tau}, \hat{d}$. The results are shown in Fig. 7 (open symbols) and illustrate that the performance of the fit differs significantly. For the discussion below, we also fitted the $\mu \mathrm{CT}$ data in the same range to the Fourier transform of the exponential correlation function

$\widetilde{C}_{\text {exp }}(k)=4 \pi \phi_{2}\left(1-\phi_{2}\right) \frac{2 \xi^{3}}{\left[1+(k \xi)^{2}\right]^{2}}$

which is a single parameter form which involves the exponential correlation length $\xi$. The results are also shown in Fig. 7 (filled symbols). For all time series of temperature gradient metamorphism (TGM-17, TGM-2, DH-1, DH-2), an intermediate drop in $R^{2}$ for both models is observed. The worst performance of the SHS model (FC sample from the DIV series) is the sample with the highest density; however, no obvious trend of $R^{2}$ with snow type or density was found. 


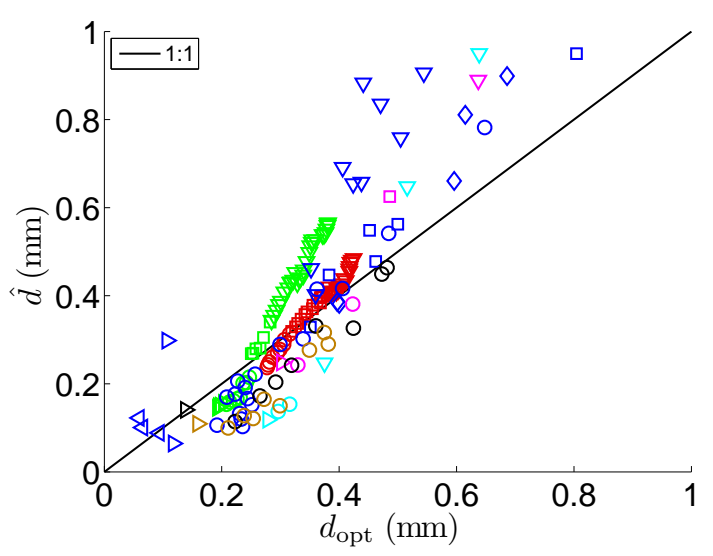

Figure 6. Scatter plot of the SHS diameter estimate $\hat{d}$ and the optical diameter $d_{\text {opt }}$. Experiment series are indicated by colors (TGM17: red, TGM-2: green, DIV: blue, DH-1: magenta, DH-2: cyan, ISO-1: black, ISO-5: brown) and snow types by markers (PP: $\triangleleft$, DF: $\triangleright$, RG: ॰, FC: $\square$, DH: $\vee$, MF: $\diamond)$. The black solid line indicates the $1: 1$ relation.

To further investigate goodness-of-fit differences of the SHS model, we analyzed the behavior of the cost function

$J(\tau, d)=\left(\sum_{i=0}^{n / 3}\left|\widetilde{C}\left(k_{i}\right)-\widetilde{C}_{\mathrm{SHS}}\left(k_{i} \mid \phi_{2}, d, \tau\right)\right|^{2}\right)^{1 / 2}$

for the SHS model $\widetilde{C}_{\text {SHS }}\left(k_{i} \mid \phi_{2}, d, \tau\right)$ from Eq. (34) which is minimized in the least-squares optimization to obtain the estimates $\hat{\tau}, \hat{d}$. The contour plot of $\log [J(\tau, d)]$ is shown in Fig. 8 for three different snow types. The three snow samples in Fig. 8 were (a) the first sample of the ISO-1 isothermal metamorphism time series (precipitation particles, PP), (b) the last sample of the ISO-1 isothermal metamorphism time series (large rounded grains, $\mathrm{RG}$ ) and (c) the last sample of the DH-2 temperature gradient metamorphism time series (depth hoar, DH). The plots indicate apparent differences in view of location and shape of the minimum with respect to snow type. For the PP example, the minimum is located close to the boundary of admissible $\tau$ values (cf. Eq. 35). In contrast, for the RG example the minimum is located well in the interior of admissible $\tau, d$ values. It is however contained in a valley almost parallel to the $\tau$ axis, indicating some degree of degeneracy of the optimal values. For the DH example the residuals are higher in magnitude and the minimum is shallower compared to the other examples. To complete the analysis of the three examples from Fig. 8, we finally plot $\mu \mathrm{CT}$ data for $\widetilde{C}(k)$ together with the SHS and the exponential model evaluated for the optimal parameters in Fig. 9.

\subsection{Analysis of grain-size scaling}

In order to assess the relevance of the grain-size scaling raised in Picard et al. (2013) we further elaborate the compar-
Table 1. Fit parameters and standard errors for a linear regression between the optimal SHS diameter and the optical diameter according to Eq. (40) carried out for subsets of the data: for individual experiment series (top) and for individual snow types (bottom).

\begin{tabular}{llcr}
\hline & Subset & $a_{1}$ & $a_{0}(\mathrm{~mm})$ \\
\hline \multirow{5}{*}{ Exp. series } & ISO-1 & $1.05 \pm 0.13$ & $-0.08 \pm 0.05$ \\
& ISO-5 & $1.04 \pm 0.16$ & $-0.11 \pm 0.05$ \\
& DIV & $1.44 \pm 0.11$ & $-0.09 \pm 0.04$ \\
& TGM-2 & $2.40 \pm 0.04$ & $-0.34 \pm 0.01$ \\
& TGM-17 & $1.47 \pm 0.03$ & $-0.16 \pm 0.01$ \\
& DH-1 & $2.38 \pm 0.12$ & $-0.58 \pm 0.05$ \\
& DH-2 & $2.03 \pm 0.21$ & $-0.40 \pm 0.09$ \\
\hline \multirow{5}{*}{ Snow type } & DF & $0.11 \pm 0.37$ & $0.14 \pm 0.07$ \\
& RG & $1.33 \pm 0.07$ & $-0.15 \pm 0.02$ \\
& DH & $1.25 \pm 0.04$ & $-0.06 \pm 0.02$ \\
& MF & $1.59 \pm 0.17$ & $-0.11 \pm 0.06$ \\
& & & $-0.33 \pm 0.10$ \\
\hline
\end{tabular}

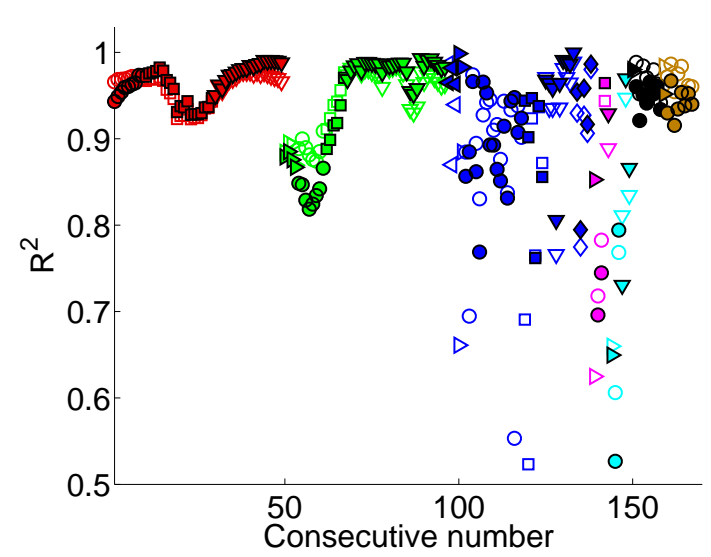

Figure 7. Coefficient of determination $R^{2}$ for the SHS model (open symbols). In addition, $R^{2}$ for the exponential model is shown (filled symbols). Experiment series are indicated by colors (TGM17: red, TGM-2: green, DIV: blue, DH-1: magenta, DH-2: cyan, ISO-1: black, ISO-5: brown) and snow types by markers (PP: $\triangleleft$, DF: $\triangleright, \mathrm{RG}: \circ, \mathrm{FC}: \square, \mathrm{DH}: \vee, \mathrm{MF}: \diamond)$.

ison of the optimal SHS diameter with the optical diameter from Fig. 6 .

As a quantitative measure, we fitted the entire data in Fig. 6 by a linear regression

$\hat{d}=a_{1} d_{\mathrm{opt}}+a_{0}$,

yielding $a_{1}=1.50$ and $a_{0}=-0.14$. If the experiment series (DIV, TGM-2, TGM-17, ISO-3, ISO-5, DH1, DH2) are individually fitted to Eq. (40) we obtain the values shown in Table 1. We also fitted Eq. (40) to each snow type class containing more than three samples. The results are also shown in Table 1.

In addition, we conducted a numerical experiment to reproduce the situation from Roy et al. (2013) where differ- 
(a)

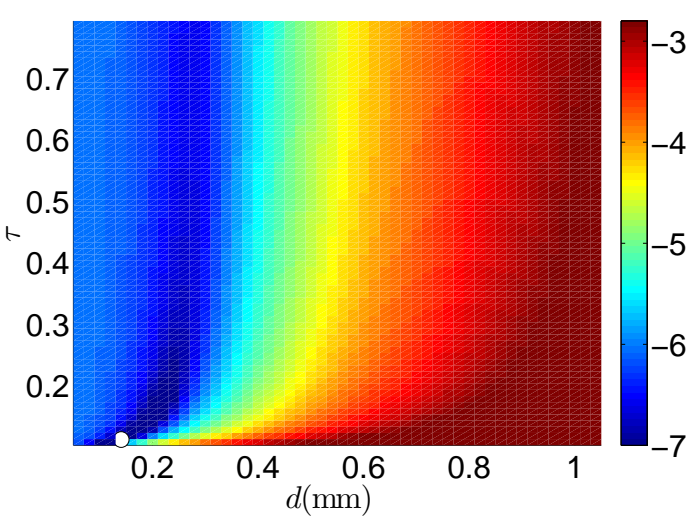

(b)

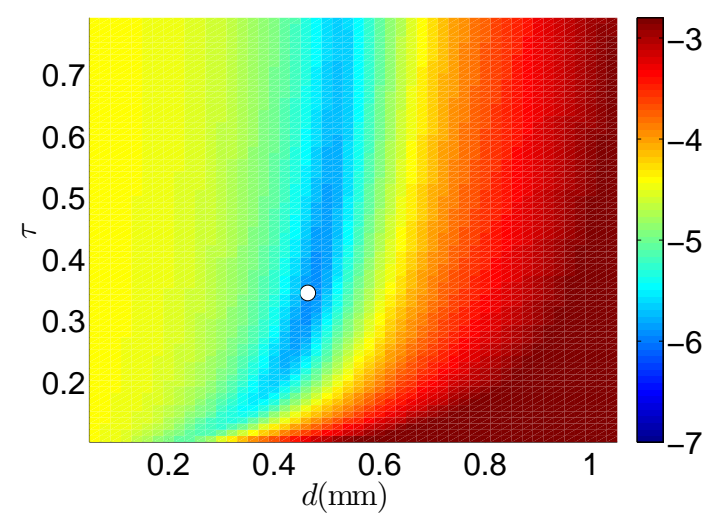

(c)

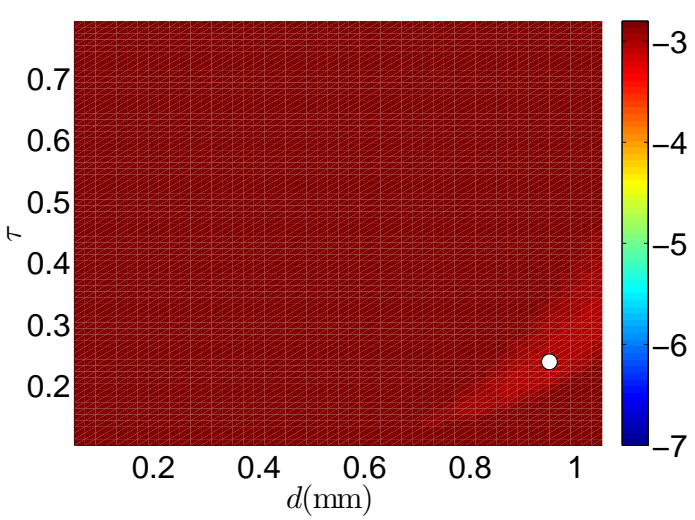

Figure 8. Contour plots of the root-mean-square error surface for different snow types. Colors show the logarithm of the sum of the squared differences between measured and parametric SHS form $\widetilde{C}(k)$ (cf. Eq. 39) as a function of $(\tau, d)$ for different examples. (a) Precipitation particles (PP). (b) Large rounded grains (RG). (c) Depth hoar (DH). The optimal values $(\hat{d}, \hat{\tau})$ for the respective sample are shown as white circles.

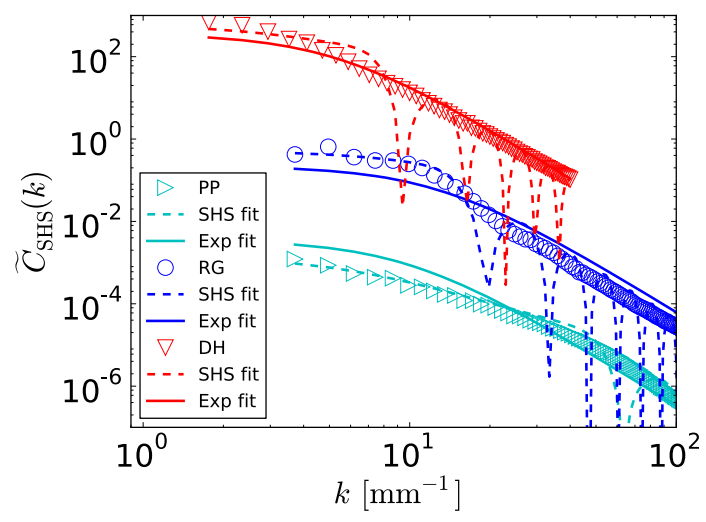

Figure 9. Comparison of the $\mu \mathrm{CT}$-derived $\widetilde{C}(k)$ data to the SHS model (Eq. 34) and the exponential model (Eq. 38). To discern individual curves, the RG and DH data were displaced vertically (by factors $10^{2}, 10^{4}$, respectively).

ent, but constant stickiness values were used. To this end we prescribed the stickiness parameters $\tau=0.13,0.44,1$, 10, 100 in the cost function Eq. (39) and conducted only a one-dimensional regression of the SHS model, now involving only the diameter as optimization parameter. This yields an optimal diameter $\hat{d}_{\tau}$ for each sample which depends on the prescribed stickiness value $\tau$. For each $\tau$ we obtain 167 pairs $\left(\hat{d}_{\tau}, d_{\text {opt }}\right)$ which are fitted to

$\hat{d}_{\tau}=b_{1} d_{\mathrm{opt}}+b_{0}$.

The results of the fit for the entire data set as a function of prescribed $\tau$ are shown in Table 2. The regression parameters are identical for $\tau=10$ and $\tau=100$ which indicates convergence to the non-sticky hard sphere model.

\subsection{Comparison of the scattering coefficient}

With the set of optimal parameters $(\hat{d}, \hat{\tau})$ from Figs. 5 and 6 we can compare the scattering coefficient and evaluate the differences between IBA and QCA-CP, when both electromagnetic models are fed with the same microstructure of SHS.

The results are shown in Fig. 10. Relative to the $1: 1$ line (full black line), a small offset is observed and the scattering coefficient from IBA is always larger than the QCA-CP counterpart. The apparent offset in the double logarithmic plot Fig. 10 is equivalent to an overall prefactor. To assess the prefactor and the deviation from $1: 1$, we evaluated the theoretical result for the ratio $r_{\mathrm{s}}$ of the scattering coefficients from Eq. (29). By computing the average volume fraction $\bar{\phi}_{2}=0.265$ of all 167 analyzed $\mu \mathrm{CT}$ samples, we can compute an average ratio $\bar{r}_{\mathrm{s}}$ between IBA and QCA-CP by inserting $\bar{\phi}_{2}$ into Eq. (29), viz $\bar{r}_{\mathrm{s}}:=r_{\mathrm{s}}\left(\bar{\phi}_{2}\right)$. This yields a value of $\bar{r}_{\mathrm{s}}=0.77$. The corresponding prediction $\kappa_{\mathrm{s}}^{\mathrm{IBA}}=\bar{r}_{\mathrm{s}} \kappa_{\mathrm{s}}^{\mathrm{QCA}-\mathrm{CP}}$ is shown in Fig. 10 as a dashed red line which fully explains the offset. 


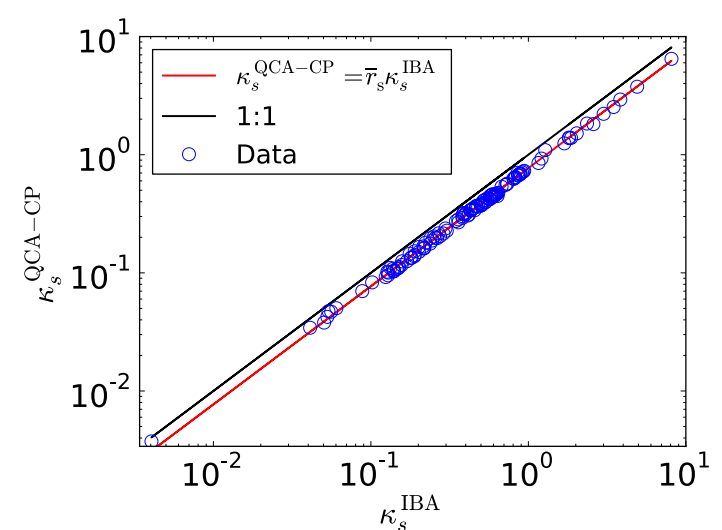

Figure 10. Scatter plot of the scattering coefficient of IBA and QCA-CP from Eqs. (28) and (22), respectively, evaluated for the optimal SHS parameters $(\hat{\tau}, \hat{d})$ retrieved from the $\mu \mathrm{CT}$ images.

\section{Discussion}

\subsection{Main results}

Three main implications can be drawn from the present work.

The first is related to the comparison of the electromagnetic models IBA used in MEMLS and QCA-CP used in DMRT-ML. By rederiving the scattering coefficient in IBA and QCA-CP and extracting the dependence on microstructure we have shown that both electromagnetic approximations involve exactly the same microstructural characteristic, namely the zero-wavevector component of the structure factor $S(0)$. This implies that the different metrics (correlation length and sphere diameter) which hitherto limited the comparison of DMRT-based and MEMLS models, is only a consequence of different microstructure descriptions and not related to fundamental differences in the respective electromagnetic theories. We derived an expression for the $k$ dependent quantity $\widetilde{C}_{\text {SHS }}\left(k \mid \phi_{2}, d, \tau\right)$ for monodisperse SHS in terms of the parameters $\phi_{2}, d, \tau$ which allows to implement exactly the same microstructure in IBA and QCA$\mathrm{CP}$. The theoretical analysis showed that if both electromagnetic models are evaluated for SHS (or any other hard sphere model), differences occur only in the zeroth-order effective dielectric constant (i.e., in the absence of scattering). The theoretical ratio of the scattering coefficient $r_{\mathrm{S}}$ was evaluated (Eq. 29, Fig. 1) and reveals only a weak dependence (maximum $30 \%$ ) on volume fraction. The theoretical ratio $r_{\mathrm{S}}$ is well-suited to explain the scatter plot for the scattering coefficient (Fig. 10) where order-of-magnitude variations are predominantly caused by variations of snow microstructure (via volume fraction, diameter and stickiness), and only marginally by the difference in the electromagnetic theories.

The second implication of the present work is related to parameter estimation itself. The closed form expression of the correlation function (or its FFT) for the monodisperse SHS model allows parameters $(\hat{d}, \hat{\tau})$ to be objectively found
Table 2. Fit parameters and standard errors for a linear regression for all samples between the optimal SHS diameter and the optical diameter if the optimization of $\hat{d}_{\tau}$ is done for prescribed $\tau$ according to Eq. (41).

\begin{tabular}{lcc}
\hline$\tau$ & $b_{1}$ & $b_{0}(\mathrm{~mm})$ \\
\hline 0.13 & $0.94 \pm 0.07$ & $0.04 \pm 0.03$ \\
0.44 & $1.43 \pm 0.09$ & $0.04 \pm 0.03$ \\
1.00 & $1.55 \pm 0.10$ & $0.03 \pm 0.03$ \\
10.0 & $1.60 \pm 0.10$ & $0.04 \pm 0.04$ \\
100.0 & $1.60 \pm 0.10$ & $0.04 \pm 0.04$ \\
\hline
\end{tabular}

for a given snow sample from a $\mu \mathrm{CT}$ image. This is of considerable interest for the stickiness since no other method to estimate this quantity from measurements is presently available. The parameter estimation showed that optimal stickiness values vary significantly (Fig. 5). This may be partially explained by the weak determination of this parameter when compared to the diameter, as illustrated by the behavior of the least-squares cost function in Fig. 8. Nevertheless, given the large sensitivity of the scattering coefficient on stickiness (Picard et al., 2013), these variations have a significant impact on the modeled electromagnetic response of snow. From the present analysis, the stickiness has to be considered as an independent parameter, which can be neither expressed in terms of the diameter (Fig. 4) nor in terms of the ice volume fraction (Fig. 5). The present work only suggests (Fig. 5) that stickiness values of snow are essentially bounded from above by the percolation line (Eq. 36) and bounded from below by the theoretical lower bound (Eq. 35) where SHS becomes physically meaningless. The variations in estimated parameters also show that the pragmatic approach of using the same stickiness value for the entire snowpack (Picard et al., 2013) is questionable. This should be considered in future use of DMRT models.

The third implication of the work is related to the applicability of what has been termed the "short range limit" in microwave models (Tsang et al., 2001, e.g., p. 504). To reveal the equivalence of the scattering coefficient between IBA and QCA-CP (Eqs. 22,28) we followed a common, but not required assumption that all relevant length scales are small compared to the wavelength. This allows to replace the $k$ dependent structure factor $S(k)$ by its value at the origin $S(0)$ in the phase function. On the other hand, the results of the fitting procedure show (Fig. 6) that the estimated stickiness values for some samples are close to the line $\tau_{\min }\left(\phi_{2}\right)$ from Eq. (35). When approaching the line, the SHS structure factor diverges (Baxter, 1968). This is a consequence of the meaning of $\tau_{\min }\left(\phi_{2}\right)$ as the coexistence line of the underlying first-order liquid-gas-phase transition in the thermodynamic framework of SHS near the critical point. Approaching the critical point is accompanied by the occurrence of density fluctuations of increasing spatial extent, causing maybe un- 
realistically large values of the scattering coefficient of IBA and QCA-CP in the short range limit (Fig. 10). In other words, estimating stickiness parameters close to the critical line renders the "short range" assumption (i.e., that all relevant length scales are small compared to the wavelength) invalid. The impact of the break-down of this assumption can be principally analyzed, e.g., in IBA, by implementing the SHS model in MEMLS where the integral over directions (Eq. 12) is computed numerically. This would allow the full $k$ dependence of $I(k)$ to be used in the phase function (Eq. 5) and the results to be compared to the short range limit $I(0)$. Similarly, QCA expressions under the long range assumption can also be obtained by numerical integration (Tsang et al., 2001, Eq. 10.2.62). This is however beyond the scope of the present work.

\subsection{Using SSA measurements to run microwave models}

One main motivation for the present work was the issue of grain-size scaling raised by Brucker et al. (2011) to relate the optical diameter of snow to microwave simulations based on SHS.

The SSA, or optical radius, of snow can be easily measured in the field and is an appealing first guess as the size parameter in DMRT-based sphere models. However, all simulations using $d=d_{\text {opt }}$ and $\tau=\infty$, i.e., non-sticky spheres (Brucker et al., 2011), underestimated the scattering coefficient. This was rectified by scaling up the sphere diameter $d$ by an empirical factor ranging from 2.3 to 3.5 . The scaling was suggested to replace the unknown dependence on stickiness and distributions of grain sizes in snow (Roy et al., 2013). The results from Fig. 5 clearly show that stickiness cannot be set to $\tau=\infty$; and thus non-sticky hard spheres are inadequate. This raises two important questions for electromagnetic modeling when accepting the necessity of stickiness and using SSA measurements. (1) Considering $\tau$ to be known, is $d=d_{\text {opt }}$ an adequate approximation? (2) Are the parameters $(\hat{\tau}, \hat{d})$ found in the present work in agreement with the grain-size scaling found in previous studies?

Figure 5 and Table 1 give a clear answer to question 1 . We always observed an affine relation $\hat{d}=a_{1} d_{\mathrm{opt}}+a_{0}$ with a slope $a_{1}$ which is (on average for the entire data set) $a_{1} \approx$ .1.50. The slope, however depends on snow type exemplified by the different subsets of snow samples used in the present analysis. We observed that the temperature gradient experiments TGM-17, TGM-2, DH-1, DH-2, which all include the formation of depth hoar (cf. Supplement in Löwe et al., 2013), lead to an apparently stronger dependence on the optical diameter (Table 1, top) when compared to the isothermal experiments, where the slope is close to unity. This impact is confirmed by restricting the relation $\hat{d}=a_{1} d_{\text {opt }}+a_{0}$ to individual snow types (Table 1, bottom), or likewise by Fig. 6 where DH samples are predominantly located above the $1: 1$ line and RG samples below.
When stickiness is not known in advance, but set to a fixed, prescribed value, our numerical experiment (Table 2) has also shown that the slope between the optical diameter and SHS diameter depends on the prescribed stickiness. A simple scaling $\hat{d}=\Phi d_{\text {opt }}$ with a grain-size scaling factor $\Phi$ is insufficient. In addition, the fit coefficient $b_{1}$ from Eq. (41) is always lower than the values for $\Phi$ found in Roy et al. (2013). This is valid, even for the non-sticky case which can be identified with $\tau=10$ or $\tau=100$ in Table 1 , as signaled by the convergence of the respective coefficients. However, a value $b_{1}=1.6$ tends to confirm a hypothesis put forward by Roy et al. (2013): they proposed that the polydisperse nature of snow would justify a scaling of 1.6 based on Jin (1994), while the stickiness would add an extra scaling by another factor of 1.6 which is the value of $b_{1}$ found here. If both effects are assumed to be independent (which is unlikely), this yields a scaling close to 2.5 found by Brucker et al. (2011) and Picard et al. (2014). Further quantitative analysis of the polydisperse SHS model is required to test this hypothesis. However, a qualitative assessment of the impact of polydispersity can be yet obtained from literature.

\subsection{Monodisperse vs. polydisperse SHS}

A peculiar feature of the monodisperse SHS correlation function $\widetilde{C}(k)$ are the oscillations in the tail for large $k$ (Fig. 3), which originate from the form factor Eq. (27). These features are clearly missing in the experimental data shown in Fig. 9, but also for any other snow sample. The oscillations are a manifestation of the monodisperse nature of SHS used here. Using polydisperse SHS with a distribution of diameters, these oscillatory features are smeared out as shown by Ginoza and Yasutomi (1999), leading to a smooth tail of $\widetilde{C}(k)$ and a more realistic appearance of the model when compared to the measurements. Furthermore, a comparison of the present results with Ginoza and Yasutomi (1999) also reveals that an increase of stickiness (increase of $\tau^{-1}$ in Fig. 3) has a similar enhancing effect on the scattering intensity for low $k$ as the increase of polydispersity (Fig. 4 in Ginoza and Yasutomi, 1999), given that the mean sphere diameter is held constant. This further supports the hypothesis on the superposition of effects on grain scaling from the previous section. This is intuitively reasonable, since both effects, polydispersity and stickiness, essentially increase the variability (i.e., fluctuations of the microscopic density) in the sample, and thereby the scattering efficiency.

The monodisperse version of SHS bears another peculiarity. In general, the Fourier transform of the correlation function must reveal the SSA in its large $k$ limit. This is known as the Porod law (Torquato, 2002) which relates the large $k$ asymptotics, $\lim _{k \rightarrow \infty} k^{4} \widetilde{C}(k)=s / 2$, to the interfacial area per unit volume $s$. This is generally valid for any two-phase system with a smooth interface, and also present in the experimental data (Fig. 3). The Porod law is mathematically equivalent to the existence 
of a cusp singularity of the real-space correlation function $C(r)$ at the origin, i.e., the existence of a small- $r$ expansion of the form $C(r) / C(0)=1-s r / 4+\mathcal{O}\left(r^{3}\right)$. However

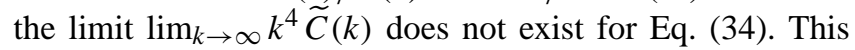
is a known, subtle problem and a consequence of monodispersity (Tomita, 1986). Thus the monodisperse SHS model bears some mathematical peculiarities which have to be examined with care.

If, however, the present method of $\mu$ CT-based parameter estimation were to be generalized to polydisperse SHS, some additional effort would be required. In the polydisperse case, a system of coupled quadratic equations must be solved to calculate the structure factor $S(k)$ according to Tsang et al. (2001, Ch. 8.4.2). This can be done only numerically in contrast to the closed-form solution for the single quadratic Eq. (32) in the monodisperse case. In addition, a generalization of Eq. (23) would be required. A similar route was suggested by Robertus et al. (1989) in the context of small angle scattering.

\subsection{Performance of the SHS model}

To assess the goodness-of-fit of monodisperse SHS, we have evaluated the coefficient of determination $R^{2}$. Large differences are observed (Fig. 7), which poses the question for which snow type SHS might be a suitable model. We also included a comparison of the $R^{2}$ for SHS with the $R^{2}$ for the exponential model which is commonly used in MEMLS. At first sight, the differences between the models seem to be smaller that sample to sample variations (Fig. 7). A discussion of small $R^{2}$ differences is subject to caution, but interestingly the temperature gradient time series (DH1, DH2, TGM2, TGM17), which evolve from decomposing particles or rounded grains into depth hoar, undergo a crossover in the relative performance of the exponential and the SHS model; while initially the SHS model is slightly superior or comparable to the exponential model, this order changes at the end of the time series. This crossover is absent for the isothermal metamorphism. This difference in performance depending on snow type is also indicated by the behavior of the cost function in Fig. 8 or by the comparison of the $\mu \mathrm{CT}$ correlation functions with both models in Fig. 9. The observed goodness-of-fit differences, depending on correlation function models and snow types, requires a more in-depth analysis in the future.

The performance of any correlation function model has to be assessed against microwave measurements which eventually decide about the quality of a particular model. We have shown that in both scattering formulations, IBA and QCA-CP, it comes down to a single microstructural quantity which must be well-predicted to describe scattering correctly in the low frequency limit. This quantity is the integral of the correlation function, or likewise, the zero-wavevector component $\widetilde{C}(0)$ of the Fourier transform. A special name has been coined for this type of parameter; it is referred to as the coarseness of the medium (Torquato, 2002). The coarseness is a single number which quantifies the residual amplitude of volume fraction fluctuations at the largest length scales.

\subsection{Reinventing the wheel: small angle scattering}

In view of the future task of finding the best microstructural model for microwave modeling, we suggest building on the exhaustive work on small angle scattering used for molecular systems. Our reanalysis has stressed that the relevant quantity in IBA and QCA-CP in the scattering coefficient for microwave modeling of snow is the Fourier transform of the correlation function which must be well-matched. This task is well-known and completely analogous to small angle scattering (SAS) of molecular systems. SAS from Xray or neutron sources has become a standard technique to characterize microstructures by fitting Fourier data (Pedersen, 1997). Indeed, the effective propagation constant and the involved length scales in molecular systems are entirely different, but the task of fitting the Fourier transform of the correlation function to a parametric model to best match the measured scattering intensity is exactly equivalent to the microwave problem in snow at low frequencies. Libraries of microstructure models (in terms of form factors, structure factors and generic forms of correlation functions for bicontinuous media) are available in free software packages, e.g., SASfit (Kohlbrecher, 2008). In principle, these packages can be applied directly to the present problem after reinterpreting (i.e., rescaling) the length scales of the $k$ axis.

\subsection{Relevance for discrete element modeling of snow}

The results about snow as a particle-based (granular) medium gained from the present work can be exploited even beyond the context of microwaves. As an example, discrete element modeling (DEM) is of special interest for snow mechanics (Johnson and Hopkins, 2005) due to the advantages in handling bond failure and the formation of new contacts under large deformations; thereby, DEM faces the same difficulty as microwave models i.e, mapping the real snow structure onto a particle-based microstructure which is in some sense equivalent to snow. Deterministic approaches, which aim to recover the exact grain structure, are very time-consuming (Hagenmuller et al., 2014). Here DEM might also benefit from a stochastic reconstruction of snow in terms of SHS, where the computational effort for the parameter estimation is in the order of seconds. For the given parameters, different realizations of the SHS model can be generated with the Monte Carlo approach described in Tsang et al. (2001). Our analysis revealed that essentially all samples lie in the percolating regime of the SHS-phase diagram (Fig. 5). This implies that the corresponding SHS structures have a static stiffness (elastic modulus) due to the percolating cluster, a prerequisite for a meaningful granular model. The interpretation of snow as a granular system via the SHS model al- 
lows, for the first time, an objective definition of a coordination number for snow as e.g., employed for a long time in snowpack models (Lehning et al., 2002). For the given parameters $\phi_{2}, \tau$ and $d$, we can use the Percus-Yevick result from Chiew and Glandt (1983) to obtain an average coordination number $n_{\mathrm{c}}=2 \phi_{2} t\left(\phi_{2}, \tau\right)$ in terms of the solution $t$ of Eq. (32). By means of the coordination number, contact with other granular approaches, e.g., for the thermal conductivity (Shertzer and Adams, 2011) or optical properties (Kaempfer et al., 2007) can be made to cross-correlate different SHSbased physical quantities.

\section{Conclusions}

We reformulated two relevant approaches to the microwave scattering coefficient of snow, namely IBA used in MEMLS and QCA-CP used in DMRT-ML, in a common microstructural framework. This revealed their quasi-equivalence when using the same microstructural, particle-based model. As an implication of the theoretical analysis, the stickiness parameter for (monodisperse) SHS can now be objectively estimated from $\mu \mathrm{CT}$ images and was found to be an essential parameter when modeling snow as a sphere assembly. Our analysis has confirmed a previous result, that the optical equivalent diameter as input for microwave models based on monodisperse SHS must be used with caution. The potential impact of polydispersity was outlined. Our preliminary goodness-offit comparison of the SHS model with the exponential model revealed an impact of snow type which must be further investigated in the future, in relation to microwave measurements, and with the help of well-established ideas from small angle scattering. Also other applications, which employ a granular picture of snow, can now take SHS with realistic parameters as input. This could be exploited to cross-correlate different physical properties and further elucidate the concept of stickiness.

Acknowledgements. We thank M. Proksch for helpful comments on the manuscript. G. Picard was sponsored by a grant from the Institut Universitaire de France and by the CNES-TOSCA SMOS project.

Edited by: R. Brown

\section{References}

Arnaud, L., Picard, G., Champollion, N., Domine, F., Gallet, J., Lefebvre, E., Fily, M., and Barnola, J.: Measurement of vertical profiles of snow specific surface area with a $1 \mathrm{~cm}$ resolution using infrared reflectance: instrument description and validation, J. Glaciol., 57, 17-29, 2011.

Baxter, R.: Percus-Yevick equation for hard spheres with surface adhesion, J. Chem. Phys., 49, 2770, doi:10.1063/1.1670482, 1968.
Brucker, L., Picard, G., and Fily, M.: Snow grain-size profiles deduced from microwave snow emissivities in Antarctica, J. Glaciol., 56, 514-526, doi:10.3189/002214310792447806, 2010.

Brucker, L., Picard, G., Arnaud, L., Barnola, J.-M., Schneebeli, M., Brunjail, H., Lefebvre, E., and Fily, M.: Modeling time series of microwave brightness temperature at Dome C, Antarctica, using vertically resolved snow temperature and microstructure measurements, J. Glaciol., 57, 171-182, 2011.

Chang, W., Tan, S., Lemmetyinen, J., Tsang, L., Xu, X., and Yueh, S.: Dense Media Radiative Transfer Applied to SnowScat and SnowSAR, IEEE J. Sel. Top. Appl., 7, 3811-3825, doi:10.1109/JSTARS.2014.2343519, 2014.

Chiew, Y. and Glandt, E.: Percolation behavior of permeable and of adhesive spheres, J. Phys. A., 16, 2599-2608, doi:10.1088/03054470/16/11/026, 1983.

Debye, P. and Bueche, A. M.: Scattering by an inhomogeneous solid, J. Appl. Phys., 20, 518-525, 1949.

Debye, P., Anderson, H., and Brumberger, H.: Scattering by an inhomogeneous solid, 2. The correlation function and its application, J. Appl. Phys., 28, 679-683, doi:10.1063/1.1722830, 1957.

Dupont, F., Picard, G., Royer, A., Fily, M., Roy, A., Langlois, A., and Champollion, N.: Modeling the Microwave Emission of Bubbly Ice: Applications to Blue Ice and Superimposed Ice in the Antarctic and Arctic, IEEE T. Geosci. Remote, 52, 6639-6651, doi:10.1109/TGRS.2014.2299829, 2014.

Fierz, C., Armstrong, R. L., Durand, Y., Etchevers, P., Greene, E., McClung, D. M., Nishimura, K., Satyawali, P. K., and Sokratov, S.: The International Classification for Seasonal Snow on the Ground, IHP-VII Technical Documents in Hydrology, 83, IACS Contribution (1), UNESCO-IHP, Paris, 2009.

Gallet, J., Domine, F., Zender, C., and Picard, G.: Measurement of the specific surface area of snow using infrared reflectance in an integrating sphere at 1310 and $1550 \mathrm{~nm}$, The Cryosphere, 3, 167-182, doi:10.5194/tc-3-167-2009, 2009.

Ginoza, M. and Yasutomi, M.: Measurable Structure Factor of a Multi-Species Polydisperse Percus-Yevick Fluid with Schulz Distributed Diameters, J. Phys. Soc. Jpn., 68, 2292-2297, doi:10.1143/JPSJ.68.2292, 1999.

Hagenmuller, P., Chambon, G., Flin, F., Morin, S., and Naaim, M.: Snow as a granular material: Assessment of a new grain segmentation algorithm, Granul. Matter, 16, 421-432, doi:10.1007/s10035-014-0503-7, 2014.

Jin, Y. Q.: Electromagnetic scattering modelling for quantitative remote sensing, World Scientific, Singapore, 1994.

Johnson, J. B. and Hopkins, M. A.: Identifying microstructural deformation mechanisms in snow using discrete-element modeling, J. Glaciol., 51, 432-442, doi:10.3189/172756505781829188, 2005.

Kaempfer, T. U., Hopkins, M. A., and Perovich, D. K.: A threedimensional microstructure-based photon-tracking model of radiative transfer in snow, J. Geophys. Res.-Atmos., 112, D24113, doi:10.1029/2006JD008239, 2007.

Kohlbrecher, J.: SASfit: A program for fitting simple structural models to small angle scattering data, Paul Scherrer Institut, Laboratory for Neutron Scattering, Villigen, Switzerland, 2008.

Lehning, M., Bartelt, P., Brown, B., Fierz, C., and Satyawali, P.: A physical SNOWPACK model for the Swiss avalanche warning 
Part II. Snow microstructure, Cold Reg. Sci. Technol., 35, 147167, 2002.

Liang, D., Xu, X., Andreadis, K. M., Josberger, E. G., and Tsang, L.: The Effects of Layers in Dry Snow on Its Passive Microwave Emissions Using Dense Media Radiative Transfer Theory Based on the Quasicrystalline Approximation (QCA/DMRT), IEEE T. Geosci. Remote, 46, 3663-3671, doi:10.1109/TGRS.2008.922143, 2008.

Löwe, H., Egli, L., Bartlett, S., Guala, M., and Manes, C.: On the evolution of the snow surface during snowfall, Geophys. Res. Lett., 34, L21507, doi:10.1029/2007GL031637, 2007.

Löwe, H., Spiegel, J. K., and Schneebeli, M.: Interfacial and structural relaxations of snow under isothermal conditions, J. Glaciol., 57, 499-510, 2011.

Löwe, H., Riche, F., and Schneebeli, M.: A general treatment of snow microstructure exemplified by an improved relation for thermal conductivity, The Cryosphere, 7, 1473-1480, doi:10.5194/tc-7-1473-2013, 2013.

Matzl, M. and Schneebeli, M.: Measuring specific surface area of snow by near-infrared photography, J. Glaciol., 52, 558-564, doi:10.3189/172756506781828412, 2006.

Mätzler, C.: Improved Born approximation for scattering of radiation in a granular medium, J. Appl. Phys., 83, 6111-6117, 1998.

Mätzler, C.: Relation between grain-size and correlation length of snow, J. Glaciol., 48, 461-466, doi:10.3189/172756502781831287, 2002.

Mätzler, C. and Wiesmann, A.: Extension of the microwave emission model of layered snowpacks to coarse-grained snow, Remote Sens. Environ., 70, 317-325, doi:10.1016/S00344257(99)00047-4, 1999.

Painter, T., Molotch, N., Cassidy, M., Flanner, M., and Steffen, K.: Instruments and Methods: Contact spectroscopy for determination of stratigraphy of snow optical grain size, J. Glaciol., 53, 121-127, 2007.

Pedersen, J.: Analysis of small-angle scattering data from colloids and polymer solutions: modeling and least-squares fitting, Adv. Colloid Interfac., 70, 171-210, doi:10.1016/S00018686(97)00312-6, 1997.

Picard, G., Brucker, L., Roy, A., Dupont, F., Fily, M., Royer, A., and Harlow, C.: Simulation of the microwave emission of multilayered snowpacks using the Dense Media Radiative transfer theory: the DMRT-ML model, Geosci. Model Dev., 6, 1061-1078, doi:10.5194/gmd-6-1061-2013, 2013.

Picard, G., Royer, A., Arnaud, L., and Fily, M.: Influence of meter-scale wind-formed features on the variability of the microwave brightness temperature around Dome C in Antarctica, The Cryosphere, 8, 1105-1119, doi:10.5194/tc-8-1105-2014, 2014.

Proksch, M., Löwe, H., and Schneebeli, M.: Density, specific surface area and correlation length of snow measured by highresolution penetrometry, J. Geophys. Res.-Earth, 120, 346-362, doi:10.1002/2014JF003266, 2015.

Pulliainen, J., Grandell, J., and Hallikainen, M.: HUT snow emission model and its applicability to snow water equivalent retrieval, IEEE T. Geosci. Remote, 37, 1378-1390, doi:10.1109/36.763302, 1999.

Robertus, C., Philipse, W. H., Joosten, J. G. H., and Levine, Y. K.: Solution of the Percus-Yevick approximation of the multicomponent adhesive spheres system applied to the small angle x-ray scattering from microemulsions, J. Chem. Phys., 90, 4482-4490, doi:10.1063/1.456635, 1989.

Rott, H., Yueh, S., Cline, D., and Duguay, C.: Cold regions hydrology high-resolution observatory for snow and cold land processes, Proc. IEEE, 98, 752-765, doi:10.1109/JPROC.2009.2038947, 2010.

Roy, A., Picard, G., Royer, A., Montpetit, B., Dupont, F., Langlois, A., Derksen, C., and Champollion, N.: Brightness Temperature Simulations of the Canadian Seasonal Snowpack Driven by Measurements of the Snow Specific Surface Area, IEEE T. Geosci. Remote, 51, 4692-4704, doi:10.1109/TGRS.2012.2235842, 2013.

Shertzer, R. H. and Adams, E. E.: Anisotropic Thermal Conductivity Model for Dry Snow, Cold Reg. Sci. Technol., 69, 122-128, 2011.

Shih, S.-E., Ding, K.-H., Kong, J. A., Yang, Y. E., Davis, R. E., Hardy, J. P., and Jordan, R.: Modeling of millimeter wave backscatter of time-varying snowcover, Prog. Electromagn. Res., 16, 305-330, 1997.

Tedesco, M. and Kim, E.: Intercomparison of Electromagnetic Models for Passive Microwave Remote Sensing of Snow, IEEE T. Geosci. Remote, 44, 2654-2666, 2006.

Tian, B., Li, Z., Zhu, Y., Chen, Q., and Wang, Y.: Quantifying inter-comparison of the microwave emission model of layered snowpacks (MEMLS) and the multilayer dense media radiative transfer theory (DMRT) in modeling snow microwave radiance, in: IEEE International Geoscience and Remote Sensing Symposium (IGARSS), Honolulu, HI, 1753-1756, doi:10.1109/IGARSS.2010.5649368, 2010.

Tomita, H.: Statistical properties of random interface system, Prog. Theor. Phys., 75, 482-495, doi:10.1143/PTP.75.482, 1986.

Torquato, S.: Random heterogeneous materials, Springer, New York, 2002.

Torquato, S. and Stell, G.: Microstructure of 2-phase randommedia, 1. The n-point probability functions, J. Chem. Phys., 77, 2071-2077, doi:10.1063/1.444011, 1982.

Torquato, S. and Stell, G.: Microstructure of two-phase random media, II. The Mayer-Montroll and Kirkwood-Salsburg hierarchies, J. Chem. Phys., 78, 3262-3272, doi:10.1063/1.445245, 1983.

Tsang, L.: Dense media radiative transfer theory for dense discrete random media with spherical particles of multiple sizes and permittivities, in: Dielectric Properties of Heterogeneous Materials, Vol. 6, chap. 5, edited by: Priou, A., Elsevier, New York, 181230, 1992.

Tsang, L. and Kong, J.: Scattering of Electromagnetic Waves, Advanced Topics, Scattering of Electromagnetic Waves, Wiley, New York, 2001.

Tsang, L., Kong, J., Ding, K., and Ao, C.: Scattering of Electromagnetic Waves: Numerical Simulations, Scattering of Electromagnetic Waves, Wiley, New York, 2001.

Tsang, L., Chen, C., Chang, A., Guo, J., and Ding, K.: Dense media radiative transfer theory based on quasicrystalline approximation with applications to passive microwave remote sensing of snow, Radio Sci., 35, 731-749, doi:10.1029/1999RS002270, 2000.

Tsang, L., Pan, J., Liang, D., Li, Z., Cline, D. W., and Tan, Y.: Modeling active microwave remote sensing of snow using dense media radiative transfer (DMRT) theory with multiple- 
scattering effects, IEEE T. Geosci. Remote, 45, 990-1004, doi:10.1109/TGRS.2006.888854, 2007.

Warren, S. G.: Optical properties of snow, Rev. Geophys., 20, 6789, doi:10.1029/RG020i001p00067, 1982.

Wiesmann, A. and Mätzler, C.: Microwave Emission Model of Layered Snowpacks, Remote Sens. Environ., 70, 307-316, doi:10.1016/S0034-4257(99)00046-2, 1999.

Xu, X., Liang, D., Tsang, L., Andreadis, K., Josberger, E., Lettenmaier, D., Cline, D., and Yueh, S.: Active Remote Sensing of Snow Using NMM3D/DMRT and Comparison With CLPX II Airborne Data, IEEE J. Sel. Top. Appl., 3, 689-697, doi:10.1109/JSTARS.2010.2053919, 2010.
Yeong, C. and Torquato, S.: Reconstructing random media, Phys. Rev. E, 57, 495-506, doi:10.1103/PhysRevE.57.495, 1998.

Zachary, C. E. and Torquato, S.: Hyperuniformity in point patterns and two-phase random heterogeneous media, J. Stat. Mech.Theory E., P12015, doi:10.1088/1742-5468/2009/12/P12015, 2009. 